\title{
The Benefits of Preoperative Tight Glycaemic Control on Postoperative Coronary Artery Bypass Grafting Outcomes in Type 2 Diabetic Patients
}

\author{
Alireza Yaghoubi ${ }^{1}$, Sohrab Negargar², Sahar Pouraghaie ${ }^{3}$, Shamsi Ghaffari ${ }^{4}$, Elgar Enamzadeh ${ }^{5}$, Naser Safaie ${ }^{6}$ \\ ${ }^{1}$ Professor of cardiovascular surgery, Rajaie Cardiovascular Medical \& Research Center, Tehran, Iran. \\ ${ }^{2}$ Professor of anaesthesiolog, Cardiovascular Research Center, Tabriz University of Medical Sciences, Tabriz, Iran. \\ ${ }^{3}$ Msc of Nursing, Cardiovascular Research Center, Tabriz University of Medical Sciences, Tabriz, Iran. \\ ${ }^{4}$ Associate professor of Paediatric cardiology, Cardiovascular Research Center, Tabriz University of Medical Sciences, \\ Tabriz, Iran. ${ }^{5}$ Assistant professor of Cardiology, Cardiovascular Research Center, Tabriz University of Medical \\ Sciences, Tabriz, Iran. ${ }^{6}$ Professor of Cardiovascular Surgery, Cardiovascular Research Center, Tabriz University of \\ Medical Sciences, Tabriz, Iran.
}

\section{ABSTRACT}

\section{BACKGROUND}

Cardiovascular diseases are one of the main causes of mortality in diabetic patients. The most important cardiac surgical procedure performed on diabetic patients is coronary artery bypass grafting surgery (CABG). This study was conducted to determine the effects of tight preoperative glycaemic control in outcomes of coronary artery bypass grafting surgery and early beneficial effects of tight glycaemic control in diabetic patients.

\section{METHODS}

In this clinical trial, 100 CABG candidates were enrolled into the study conducted in Shahid Madani Hospital, Tabriz, Iran. Patients were divided into control group lacking regular blood glucose control, and case group with regular blood glucose control.

\section{RESULTS}

The mean glucose levels in case and control groups before surgery was $183.34 \pm$ 38.72 vs $230.75 \pm 91.47 \mathrm{mg} / \mathrm{dL}$, respectively ( $\mathrm{p}=0.005$ ); during surgery, mean glucose level was $138.67 \pm 33.41$ vs $255.27 \pm 54.69 \mathrm{mg} / \mathrm{dL}(\mathrm{P}<0.001)$. In a three-day period after surgery, mean glucose level was $119.79 \pm 9.30$ vs $209.75 \pm 28.74 \mathrm{mg} / \mathrm{dL}$ ( $\mathrm{p}<0.001)$ on the first day, $122.94 \pm 9.50$ vs $183.72 \pm 24.18 \mathrm{mg} / \mathrm{dL}(\mathrm{p}<.001)$ on the second day of surgery and $136.30 \pm 14.84 ; 200.03 \pm 41.70 \mathrm{mg} / \mathrm{dL}(\mathrm{p}<0.001)$ on the third day. Consumption of oral hypoglycaemic agents $(p=0.31)$, NYHA classification $(p=0.56)$, preoperative ejection fraction $(E F)(\%)(p=0.08)$, type of surgery $(p=0.09)$ were statistically similar between groups.

\section{CONCLUSIONS}

Tight blood glucose control is effective in controlling hyperglycaemia, decreasing hospitalization period of patients, and reducing hospital costs in diabetic patients undergoing CABG.

\section{KEY WORDS}

Diabetes, Coronary Artery Bypass Grafting Surgery, Glycaemic Control

\author{
Corresponding Author: \\ Naser Safaie, \\ Cardiovascular Research Center, \\ Tabriz University of Medical Sciences, \\ Tabriz, Iran. \\ E-mail: safaie.n@yahoo.com
}

DOI: 10.14260/jemds/2019/575

Financial or Other Competing Interests: None.

How to Cite This Article:

Yaghoubi A, Negargar S. Pouraghaie S. et al. The benefits of preoperative tight glycaemic control on postoperative coronary artery bypass grafting outcomes in type 2 diabetic patients. J. Evolution Med. Dent. Sci. 2019;8(33):2644-2648, DOI: $10.14260 /$ jemds $/ 2019 / 575$

Submission 07-04-2019, Peer Review 01-07-2019, Acceptance 07-07-2019, Published 19-08-2019. 


\section{BACKGROUND}

Cardiovascular disease (CVD) is the leading cause of mortality for 17.5 million people in the world, which is about 30 percent of total mortality rate. Of this number, 7.6 million people suffer coronary artery disease (CAD).(1-4) CAD is a major cause of mortality, morbidity, and disability. It constitutes nearly 50 percent of all deaths annually. CAD is recognized by the presence of atherosclerosis in the epicardial coronary arteries.(2)

Diabetes mellitus (DM) as well as stress hyperglycaemia in non-diabetic individuals are predictors of morbidity and/or mortality in patients referred to the hospital with myocardial infarction or unstable ischemic syndromes, and those enduring a variety of surgical procedures. (5-9) The risk of future cardiovascular events is increased with poor glycaemic control. Prospective studies of patients with type 1 and type 2 DM have proved that the incidence of vascular complications is decreased by lifestyle modifications or medications that decrease blood glucose concentrations.(10) Hypoglycaemia is also a risk for tight insulin therapy and glycaemic control. Patients, generally anesthetized in a high workload environment, may have increased rates of silent episodes of hypoglycaemia.(11)

Accordingly, presence of DM not only affects the success of surgeries, but also affects the survival rate and quality of life after surgery. One of the most important therapies for CAD is coronary artery bypass grafting (CABG) surgery. CABG surgery is a known reason for occurrence of glucose metabolism changes, glucose intolerance, resistance against insulin, and stress hyperglycaemia.(12-16)

In study by Reyden et al on the effect of preoperative blood glucose level on surgery results, uncontrolled blood glucose level was shown to increase complications such as myocardial infarction, acute coronary syndrome, and stroke in preoperative stage as well as wound infection and shock in postoperative stage. CAD is associated with the highest rate of mortality due to diabetes. Mortality rate is especially higher in women as a result of glycometabolic imbalance.(13)

In a study by Vlasselaers et al on the effect of tight glycaemic control in neonatal heart surgery, it was concluded that in neonates experiencing cardiac surgery, intraoperative and postoperative TGC protects the myocardium and lowers the inflammatory response. It seems that this is not mediated by an early direct insulin signalling effect, but it is possibly because of independent effects of preventing hyperglycaemia during reperfusion. $(17,18)$

According to the studies mentioned above, DM with its wide range of metabolic changes not only affects cardiovascular status, but also may affect procedure outcomes. This study was therefore conducted to determine whether tight preoperative glycaemic control in diabetic CABG patients would improve preoperative outcomes. We also investigated the role of early beneficial effects of tight glycaemic control on survival.

\section{METHODS}

This case-control study was conducted in Shahid Madani hospital, Tabriz, Iran, which is the main referral cardiology hospital in northwest of Iran. One hundred patients from the cardiac surgery ward and intensive care unit (ICU) were included in this study. Inclusion criteria were as follows-

- Patients undergoing on-off pump CABG surgery for the first time.

- $\quad$ Patients diagnosed with Type 2 diabetes in pre-admission stage.

- Patients who diagnosed as type-2 diabetic patients in admission stage of hospital with fasting blood glucose test, postprandial blood glucose test, and oral glucose tolerance test (OGTT) base on definition of Type 2 diabetes in American Diabetes Association.

- Patients who were interested in taking part in the study.

Patients undergoing second on-off pump CABG surgery, patients with type- 1 diabetes, creatinine level more than 2 $\mathrm{mg} / \mathrm{dL}$, or those who were expected to have other surgery in addition to CABG, were excluded from this study.

Patients were randomly divided into two 50-person groups (intervention group; with regular blood glucose control, and control group; without regular blood glucose control) using Rand List software (Version 1.2, DatInf GmbH) and applying random specific blocks (10 units).

First examined outcome of tight control glycaemic status was blood glucose level; Patients in the intensive treatment group received a continuous intravenous insulin infusion in ICU and during the surgery. 250 units of Novolin R (Novo Nordisk, Princeton, New Jersey) in $250 \mathrm{~mL}$ of $0.9 \%$ sodium chloride were administered, when the blood glucose levels passed $5.6 \mathrm{mmol} / \mathrm{L}(100 \mathrm{mg} / \mathrm{dL})$. We adjust the infusions to keep blood glucose levels in the range of $4.4(80 \mathrm{mg} / \mathrm{dL})$ to 5.6 $\mathrm{mmol} / \mathrm{L}(100 \mathrm{mg} / \mathrm{dL})$. This control was maintained by patients' cardiologist. We adjusted the dose based on a standardized algorithm used by anaesthesiologists.

Patients in the conventional treatment group did not receive insulin during surgery unless their glucose levels were more than $11.1 \mathrm{mmol} / \mathrm{L}(200 \mathrm{mg} / \mathrm{dL})$. When glucose concentration was between $11.1 \mathrm{mmol} / \mathrm{L}(200 \mathrm{mg} / \mathrm{dL})$ and $13.9 \mathrm{mmol} / \mathrm{L}(250 \mathrm{mg} / \mathrm{dL})$, patients received an intravenous bolus of 4 unit's insulin each hour until the glucose concentration was lower than $11.1 \mathrm{mmol} / \mathrm{L}$ ( $200 \mathrm{mg} / \mathrm{dL}$ ). If the intraoperative glucose concentration exceeded 13.9 $\mathrm{mmol} / \mathrm{L}(250 \mathrm{mg} / \mathrm{dL})$, an intravenous infusion of insulin was given to patients. This was continued until the glucose level was reduced to an amount less than $8.3 \mathrm{mmol} / \mathrm{L}(150 \mathrm{mg} / \mathrm{dL})$.

In both study groups, arterial plasma glucose concentration was measured every 30 minutes, beginning right before anaesthetic induction using hexokinase method on a Double $\mathrm{P}$ Modular System (Roche Diagnostics, Indianapolis, Indiana). Intraoperative procedures, including cardiopulmonary bypass, monitoring, laboratory testing, and treatment were left to the discretion of anaesthesiologists and cardiac surgeons. There was not any standard protocol to monitor and manage intraoperative potassium levels.

\section{Statistical Analysis}

The obtained data were statistically analysed using statistical software of SPSS version 15.0. Normality of data distribution was assessed by Kolmogorov-Smirnov test. Categorical variables were presented as frequency and percentages. 
Continuous variables were shown as mean (Standard deviation). Categorical variables were compared using chisquare test or Fisher exact test as appropriate. Continuous variables were compared between groups using independent samples t test and one-way ANOVA. Repeated measurement of ANOVA was used to investigate the changes of blood glucose during study period. ICU stay and hospitalization duration was analysed using Mann-Whitney U test. P-value less than 0.05 was considered statistically significant.

\section{Ethical Considerations}

On admission day, after necessary explanations about the study plan and acquiring informed consent, patients were included in the study. It should be mentioned that patients' enrolment in the study was totally optional and patients were able to leave the study at any time. All stages of study protocol were confirmed by Ethical Committee (8815- 88.3.11) of Tabriz University of Medical Sciences based on Declaration of Helsinki.

\section{RESULTS}

Demographic characteristics of both groups were presented in Table 1. According to this table, sex, age and BMI difference between two groups, was not statistically significant.

The results of comparing the clinical characteristics of type 2 diabetes in the two groups are presented in Table 2 . It can be seen that only Duration of diabetes diagnosis has a significant difference between the two groups $(\mathrm{p}<0.001)$. Consumption of oral hypoglycaemic agents as well as regular or NPH insulin was similar between groups. NYHA classification $(p=0.56)$, preoperative ejection fraction $(E F)(\%)(p=0.08)$, type of surgery $(p=0.09)$ were statistically similar between groups.

Results of blood glucose measurement in different stages of hospitalization are shown in Figure 1. It can be seen that the glucose level was significantly different between the two groups during the first 24 hours before surgery $(\mathrm{p}<0.05)$. Also, there was a significant difference between the two groups during the surgical stage $(p=0.005)$, the first 24 hours after surgery $(p<0.001)$, the second day after surgery $(p=0.001)$ and the third day after surgery $(\mathrm{p}<0.001)$.

Mean hospitalization duration of patients including preoperative, intraoperative, and postoperative stages of cardiac surgery were compared between two groups using Mann-Whitney U test. Mean duration of hospitalization was significantly lower in case group vs. control group (141:58 \pm $36: 26: 19 ; 221: 55 \pm 109.16$ respectively) $(\mathrm{p}<0.001)$.

Mean duration of ICU stay after cardiac surgery were compared in two groups using Mann-Whitney U test and it was significantly different in two groups (63:36:22 $\pm 28: 24: 30$ and 45:38:34 $\pm 12: 48: 22$, $\mathrm{p}=0.004$ ).

Two groups were significantly different regarding the hospital costs including pre, intra, and postoperative stages $(612.65 \pm 173.59$ and $421.61 \pm 60.53, \mathrm{p}<0.001)$.

Time spent by nursing staff for controlling blood glucose in pre, intra, and postoperative stages was significantly different between two groups $(136.73 \pm 71.11$ and $159.31 \pm 22.33 \mathrm{p}=$ 0.003).

\begin{tabular}{|c|c|c|c|c|}
\hline \multicolumn{2}{|r|}{$\begin{array}{c}\text { Demographic Characteristics } \\
\text { of Patients }\end{array}$} & $\begin{array}{r}\text { Intervention } \\
\text { Group }(\mathrm{N}=50)\end{array}$ & $\begin{array}{l}\text { Control Group } \\
(\mathrm{N}=\mathbf{5 0})\end{array}$ & $\mathbf{p}$ \\
\hline & Age (years) & $60.28 \pm 8.38$ & $59.38 \pm 9.01$ & 0.60 \\
\hline \multicolumn{2}{|r|}{ Sex $(\%)$} & \begin{tabular}{|c|}
28 female $(56 \%)$ \\
22 male $(44 \%)$
\end{tabular} & $\begin{array}{c}26 \text { female }(52 \%) \\
24 \text { male }(48 \%)\end{array}$ & 0.84 \\
\hline \multirow{3}{*}{$\underset{*}{\stackrel{0}{3}}$} & Normal (18.5-24.9) & $18(36 \%)$ & $15(30 \%)$ & \multirow{3}{*}{0.08} \\
\hline & $\begin{array}{l}\text { Overweight }(25-29.9) \\
\text { Obese }(30-39.9)\end{array}$ & $26(52 \%)$ & $34(68 \%)$ & \\
\hline & Severe obesity $(>40)$ & $6(12 \%)$ & $1(2 \%)$ & \\
\hline \multicolumn{5}{|c|}{$\begin{array}{c}\text { Table 1. Comparison of Demographic Characteristics } \\
\text { in the Two Groups }\end{array}$} \\
\hline \multicolumn{5}{|c|}{$\begin{array}{c}\text { BMI: Body Mass Index (WHO, Global Database on Body Mass Index. } \\
\text { *- Minimum BMI among patients was } 18.5 .\end{array}$} \\
\hline
\end{tabular}

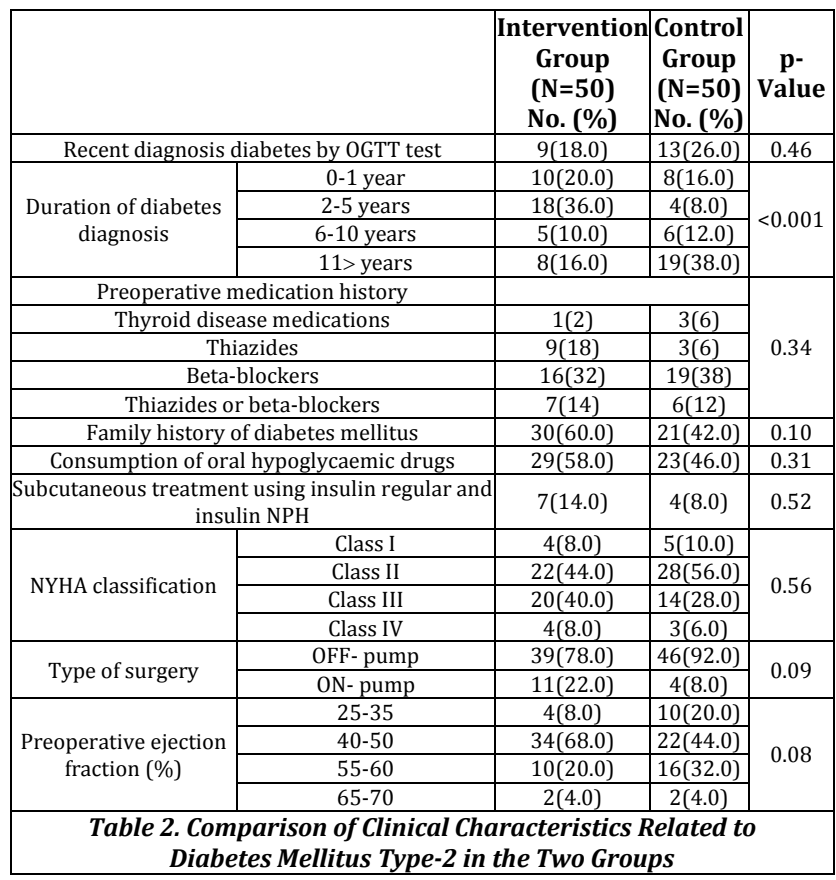

\section{DISCUSSION}

In this study, type 2 diabetic patients undergoing CABG surgery were similar and homogeneous in terms of demographic characteristics, diabetes mellitus type-2 characteristics, clinical characteristics, CAD, and coronary artery revascularization. Regular controlling of blood glucose in patients suffering type-2 diabetes in preoperative, intraoperative, and postoperative stages of CABG causes a decrease in blood glucose level. This strategy also decreases duration of hospital and ICU stay, hospital and treatment cost, as well as incidence of hyperglycaemia during hospitalization period.

In a study on diabetic patients enduring coronary artery bypass graft, it was found that individuals with diabetes, who underwent CABG, were at higher risk of adverse cardiovascular and septic complications. Diabetic individuals undergoing $C A B G$ in a large tertiary care center died either in intraoperative stage or within the first postoperative 24 hours. Some also suffered nonfatal stroke or myocardial infarction, developed a septic complication (Most frequently wound infection), or died during their hospitalization period and also stayed at hospital for a longer time,(5) our findings verified this study. 

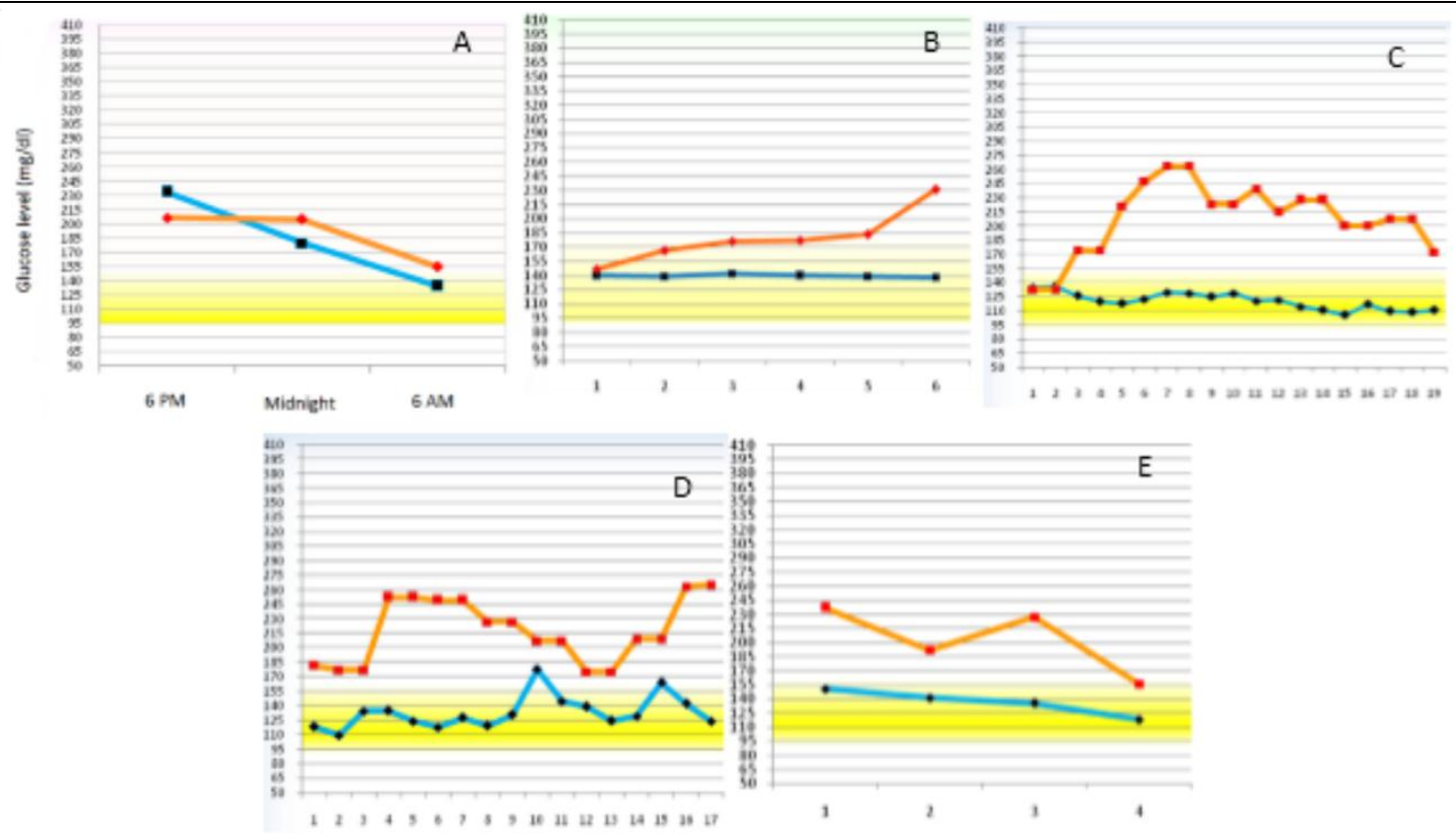

\footnotetext{
A. Before Operation

B. During Operation (every hour, six hours)

C. First day after operation in intensive care unit

D. Second day after operation in intensive care unit

E. Third day after operation in intensive care unit
}

Figure.1 Blood Glucose Level during the Study

Another study was designed to examine whether continuous insulin infusion reduces mortality in patients with diabetes undergoing coronary artery bypass grafting. It was concluded that tight control glycaemic condition by insulin infusion reduced postoperative mortality. Insulin infusions in DM patients experiencing CABG diminish mortality and eliminate the incremental increase in risk-adjusted mortality previously attributed to DM, also it led to less hospitalization period,(19) which is similar to results of our study.

In a prospective cohort study which was designed to determine the association of poor intraoperative glycaemic control with increased intra-hospital morbidity and hospitalization duration, it was concluded that in treated diabetic patients, who underwent on-pump cardiac surgery, despite aggressive insulin therapy, the intraoperative glycaemic control could be reduced difficulty. In these patients, the occurrence of a poor intraoperative glycaemic control was correlated with a worsened hospital outcome (20); This is similar to results of current study.

Not also in CABG patients but also in critically ill patients the use of exogenous insulin to maintain blood glucose at the level lower than $110 \mathrm{mg} / \mathrm{dL}$ reduces morbidity and mortality amongst critically ill patients in the surgical intensive care unit, irrespective of whether they have a history of diabetes so the hospitalization duration and costs decreases. (21)

In a study on estimating the magnitude of association between intraoperative hyperglycaemia and preoperative outcomes in patients who underwent cardiac surgery it was concluded that intraoperative hyperglycaemia is an independent risk factor for complications like death, longer hospitalization duration and increased costs after cardiac surgery, (22) this is similar to results of our study.

Using subcutaneous insulin protocol in preoperative, intraoperative and postoperative ICU and column algorithm of intravenous insulin infusion and subcutaneous insulin protocol in postoperative stage nurses with lower dependence to doctors could effectively control and lead blood glucose level to target blood glucose level through regular glycaemic control, so tight blood glucose control is advised for controlling hyperglycaemia, decreasing hospitalization period of patients, and reducing hospital costs and nursing time which are short term outcomes of tight blood glucose control.

\section{CONCLUSIONS}

Tight blood glucose control is effective in controlling hyperglycaemia, decreasing hospitalization period of patients, and reducing hospital costs in diabetic patients undergoing CABG.

\section{REFERENCES}

[1] Kuper H, Marmot M, Hemingway H. Systematic review of prospective cohort studies of psychosocial factors in the etiology and prognosis of coronary heart disease. Seminars in Vascular Medicine 2002;2(3):267-314.

[2] Writing Group Members, Loyd-Jones D, Adams RJ, et al. Heart disease and stroke statistics-2010 update: a report from the American Heart Association. Circulation 2010;121(7):e46-e215.

[3] Lloyd-Jones D, Adams R, Carnethon M, et al. Heart disease and stroke statistics-2009 update: a report from the American Heart Association Statistics Committee and Stroke Statistics Subcommittee. Circulation 2009;119(3):e21-181. 
[4] Akbarzadeh F, Shadravan S, Ghorbanian M, et al. Double left anterior descending coronary artery originating from left main coronary stem and right coronary artery. Journal of Cardiovascular and Thoracic Research 2013;5(2):73-5.

[5] McAlister FA, Man J, Bistritz L, et al. Diabetes and coronary artery bypass surgery: an examination of perioperative glycaemic control and outcomes. Diabetes Care 2003;26(5):1518-24.

[6] Capes SE, Hunt D, Malmberg K, et al. Stress hyperglycaemia and increased risk of death after myocardial infarction in patients with and without diabetes: a systematic overview. The Lancet 2000;355(9206):773-8.

[7] Micha R, Wallace SK, Mozaffarian D. Red and processed meat consumption and risk of incident coronary heart disease, stroke, and diabetes mellitus: a systematic review and meta-analysis. Circulation 2010;121(21):2271-83.

[8] Hansson GK. Inflammation, atherosclerosis and coronary artery disease. New England Journal of Medicine 2005;352(16):1685-95.

[9] Naghavi-Behzad M, Alizadeh M, Azami S, et al. Risk factors of congenital heart diseases: a case-control study in Northwest Iran. Journal of Cardiovascular and Thoracic Research 2013;5(1):5-9.

[10] LeRoith D, Rayfield EJ. The benefits of tight glycaemic control in type 2 diabetes mellitus. Clinical Cornerstone 2007;8(Suppl 7):S19-S29.

[11] Van den Berghe G, Wouters PJ, Bouillon R, et al. Outcome benefit of intensive insulin therapy in the critically ill: Insulin dose versus glycaemic control. Critical Care Medicine 2003;31(2):359-66.

[12] Inman BA, Sauver JLS, Jacobson DJ, et al. A populationbased, longitudinal study of erectile dysfunction and future coronary artery disease. Mayo Clinic Proceedings 2009;84(2):108-13.

[13] Davies SW, O'Neal WT, Sawyer RG, et al. A comparison of the long-term survival of 306,868 patients with multivessel coronary artery disease undergoing cabg and pci: a meta-analysis of retrospective studies. Journal of the American College of Cardiology 2013;61(Suppl 10):E1214.
[14] Harrington RA. ACP Journal Club. PCI using drug-eluting stents had higher mortality than CABG in diabetes and multivessel CAD. Annals of Internal Medicine 2013;158(6):JC8.

[15] Ward RJ. Diagnosed diabetes and in-hospital mortality after coronary artery bypass grafting. Emory University 2012.

[16] Azami-Aghdash S, Ghaffari S, Sadeghi-Bazargani H, et al. Developing indicators of service quality provided for cardiovascular patients hospitalized in cardiac care unit. Journal of Cardiovascular and Thoracic Research 2013;5(1):23-8.

[17] Vlasselaers D, Mesotten D, Langouche L, et al. Tight glycaemic control protects the myocardium and reduces inflammation in neonatal heart surgery. The Annals of Thoracic Surgery 2010;90(1):22-9.

[18] Aronson D, Edelman ER. Role of CABG in the management of obstructive coronary arterial disease in patients with diabetes mellitus. Current Opinion in Pharmacology 2012;12(2):134-41.

[19] Furnary AP, Gao G, Grunkemeier GL, et al. Continuous insulin infusion reduces mortality in patients with diabetes undergoing coronary artery bypass grafting. The Journal of Thoracic and Cardiovascular Surgery 2003;125(5):1007-21.

[20] Ouattara A, Lecomte P, Le Manach Y, et al. Poor intraoperative blood glucose control is associated with a worsened hospital outcome after cardiac surgery in diabetic patients. Anesthesiology 2005;103(4):687-94.

[21] Van Den Berghe G, Wouters P, Weekers F, et al. Intensive insulin therapy in critically ill patients. New England Journal of Medicine 2001;345(19):1359-67.

[22] Gandhi GY, Nuttall GA, Abel MD, et al. Intraoperative hyperglycaemia and perioperative outcomes in cardiac surgery patients. Mayo Clinic Proceedings 2005;80(7):862-6. 